\title{
3d Beam Reconfigurable THz Antenna with Graphene-Based High-Impedance Surface
}

\author{
Caixia Wang ${ }^{1}{ }^{(0)}$, Yuan Yao ${ }^{1, *}$, Junsheng $\mathrm{Yu}^{1}$ and Xiaodong Chen ${ }^{2}$ \\ 1 School of Electronic Engineering, Beijing University of Posts and Telecommunications, Beijing 100876, China; \\ wcx1992@bupt.edu.cn (C.W.); jsyu@bupt.edu.cn (J.Y.) \\ 2 School of Electronic Engineering and Computer Science, Queen Mary, University of London, \\ London E1 4NS, UK; xiaodong.chen@qmul.ac.uk \\ * Correspondence: yaoy@bupt.edu.cn
}

Received: 17 September 2019; Accepted: 4 November 2019; Published: 5 November 2019

\begin{abstract}
In this letter, a novel 3D multi-beam reconfigurable THz loop antenna capable of steering its main beam in the semi-sphere space $\left(\theta \in\left\{0^{\circ}, \pm 5^{\circ}, \pm 10^{\circ}\right\} ; \varphi \in\left\{0^{\circ}, 30^{\circ}, 60^{\circ}, 90^{\circ}, 120^{\circ}, 150^{\circ}\right\}\right)$ is presented. The antenna is based on a switchable circular high impedance surface (HIS) using the graphene-metal hybrid approach. The effect of gate voltage on the conductivity of graphene and the switchable reflection characteristic of the graphene-based HIS are combined in the design. Changing the chemical potential of different graphene-based HIS units can effectively adjust the beam direction. The performance of the antenna is analyzed through its reflection coefficients and gain radiation patterns, and simulated results show that the maximum gain can reach $3.23 \mathrm{dBi}$ at $0.5 \mathrm{THz}$.
\end{abstract}

Keywords: beam reconfigurable; THz antenna; graphene; high-impedance surface

\section{Introduction}

Due to the rapid development of wireless communication, reconfigurable antennas have attracted much attention in recent years [1]. Frequency reconfigurable antennas allow frequency switching and dynamic spectrum allocation [2], while pattern reconfigurable antennas can be used to filter in-band interference and increase the channel capacity [3-5]. In the microwave frequency band, frequency reconfigurability in antennas can be realized by employing switches, such as PIN diodes, microelectromechanical systems (MEMS), optically controlling microwave switches, and so on [6]. However, in the terahertz $(\mathrm{THz})$ frequency range, these switches are difficult to design.

Graphene is a two-dimensional (2D) planar structure built up by carbon atoms in a honeycomb lattice, which possesses many unique chemical, thermal, electronic, and optical characteristics [7-9]. In particular, the surface conductivity can be dynamically altered over a wide range by changing bias voltage, which makes it a promising candidate for $\mathrm{THz}$ and infrared frequency bands, such as antennas [6,10,11], polarizers [12], phase shifters [13], switches [14], some THz modulators [15], demodulators [16], mixers [17], and THz receivers [18]. In References [10,11], graphene works as a radiation part of the antennas because of the capability of transmitting the surface plasmon polariton (SPP) waves. However, its small electrical size and high ohmic loss leads to low radiation efficiency. To improve the radiation efficiency, graphene-metal hybrid structures have been designed $[19,20]$. A THz beam reconfigurable loop antenna enhancing the radiation efficiency by graphene-metal hybrid is proposed in Reference [19]. In Reference [20], a beam-scanning microstrip quasi-Yagi-Uda antenna working at $2.6 \mathrm{THz}$ is presented, and the $H$-plane radiation pattern can be deflected from $-70^{\circ}$ to $70^{\circ}$ by controlling the bias voltage of graphene. This balances the efficiency and miniaturization of graphene-based antenna. 
Furthermore, the high-impedance surface (HIS) is a periodical structure with the ability to reflect electromagnetic waves in phase in the resonance frequency bands [21-26]. In many RF devices, such as filters, antennas, and absorbers, HIS has been employed to enhance gain and efficiency. In Reference [22], the characteristics of a dipole over PEC or PMC layers and the HIS are compared, and a low-profile antenna with directional pattern and higher impedance bandwidth is achieved by using HIS. To further improve the bandwidth and radiation performance, Mikal Askarian Amiri et al. proposed a circular symmetric HIS in Reference [23], where cylindrical TEM ${ }^{\mathrm{z}}$ wave illumination is used to characterize the reflection characteristics of the surface. When the HIS is used as a ground plane for loop antennas, the operational bandwidth is about $10 \%$ wider than that of the rectangular surface. Recently, graphene-based HIS with controllable operation modes have been widely studied. Yi Huang et al. have presented a beam-steering THz antenna with graphene-based HIS, and the radiation beam can vary in the range of $\pm 30^{\circ}$ [27]. Besides this, a graphene-based frequency and beam reconfigurable leaky-wave antenna in $\mathrm{THz}$ frequency band was achieved in Reference [28]. In this design, a graphene sheet is used as a tuning part of the HIS that acts as the ground plane of such 2-D LWA (Leaky wave antenna), and by adjusting the graphene conductivity, the reflection phase of the HIS can be altered effectively.

In addition, the simultaneous beam steering in multiple different planes is an interesting and challenging area to be explored. In this letter, a novel 3D multi-beam reconfigurable $\mathrm{THz}$ antenna is presented to provide improved performance in wireless systems. The antenna consists of a switchable circular symmetric HIS based on graphene and a loop antenna over it. Changing the DC bias of different graphene-based HIS units can effectively adjust the beam direction. Simulation results show that twenty-five steerable beam directions in the semi-sphere space $\left(\theta \in\left\{0^{\circ}, \pm 5^{\circ}, \pm 10^{\circ}\right\} ; \varphi \in\left\{0^{\circ}, 30^{\circ}\right.\right.$, $\left.\left.60^{\circ}, 90^{\circ}, 120^{\circ}, 150^{\circ}\right\}\right)$ are achieved.

\section{Antenna Design}

\subsection{Switchable Graphene-Based High Impedance Surface (HIS)}

It is well known that graphene can be modeled as an infinitely thin surface, which is characterized by a surface conductivity [7-10]. According to Kubo's formula, in the low THz range ( $f \in[0.3,1] \mathrm{THz})$, and at room temperature $\mathrm{T}=300 \mathrm{~K}$, the graphene surface conductivity can be approximated as [13]:

$$
\sigma=\frac{2 e^{2} K_{B} T}{\pi h^{2}(\mathrm{j} \omega+2 \Gamma)} \ln \left[2 \cosh \left(\frac{\mu_{c}}{2 K_{B}}\right)\right]
$$

where $e$ is the electron charge, $k_{B}$ is the Boltzmann constant, $T$ is the temperature, $\hbar=h /(2 \pi)$ is the reduced Plank constant, $\omega$ is the angular frequency, $\Gamma=1 /(2 \tau)$ is the scattering rate, $\tau$ is the relaxation time, and $\mu_{c}$ is graphene chemical potential. Graphene chemical potential $\mu_{c}$ can be tuned by applying a transverse electric field. This makes the adjustability of graphene surface conductivity feasible. According to Reference [20], the external electric field, DC bias V, can be calculated by

$$
V=V_{0}+\frac{t e \mu_{c}^{2}}{\varepsilon_{0} \varepsilon_{r} \pi h^{2} v_{F}^{2}}
$$

where $V_{0}$ is the voltage compensation dependent on the chemical doping, $t$ and $\varepsilon_{r}$ are the thickness and relative permittivity of the dielectric between graphene and electrode, respectively, and $v_{F}=9.5 \times 10^{5} \mathrm{~m} / \mathrm{s}$ is the Femi velocity. By loading the graphene units under the circular patch of the HIS, the operative modes of the proposed graphene-based HIS units are expected to be controlled due to the alterable surface conductivity of the graphene. When the chemical potential of a graphene-based HIS unit $\mu_{c}$ equals $0.3 \mathrm{eV}$, the bias voltage $V=3.48 \mathrm{~V}$ can be calculated by Equation (2), considering $V_{0}=0, t=10 \mathrm{~nm}$ [20], and $\varepsilon_{r}=3.8$, the graphene can be considered as a dielectric-like material, so the graphene layer has a negligible influence on the HIS performance. At the resonant 
frequency, the reflection phase crosses through $0^{\circ}$ and the amplitude of reflection coefficient reaches its minimum [29]. The proposed HIS unit is still in the high-impedance state. On the contrary, when the chemical potential $\mu_{\mathrm{c}}=0 \mathrm{eV}$, the graphene switched to a conductor-like material [29], and efficiently block the electromagnetic wave penetrating it. There is no resonance within the frequency range of interest, and the HIS unit is switched to low-impedance state.

\subsection{Antenna Structure with the Graphene-Based High Impedance Surface (HIS)}

Figures 1 and 2 show the configuration of the loop antenna with the graphene-based HIS. The circular HIS is based on $\mathrm{SiO}_{2}$ substrate with relative permittivity $\varepsilon_{r}=3.8$ and a thickness $H_{1}=1 \mu \mathrm{m}$, the bottom surface of which is covered with a gold sheet. The top surface of the dielectric is the graphene-based HIS units that have periodic gaps in the angular direction. The number of cells $N$ is 12. The angle of the graphene unit cells is the sum of $\alpha$ and $\beta$, and the graphene-patch angle $\alpha$ and the gap angle $\beta$ of the unit are $27^{\circ}$ and $3^{\circ}$, respectively. The HIS over the graphene consists of four single rings. The radius of the inner ring $\mathrm{r}$ is $25 \mu \mathrm{m}$, and the radial patch length and the radial gap length of each ring are $w_{\mathrm{r}}=40 \mu \mathrm{m}$ and $g_{\mathrm{r}}=1 \mu \mathrm{m}$. The patch angles of each single ring are optimized to $27^{\circ}$, $23.4^{\circ}, 21.6^{\circ}$, and $20.5^{\circ}$, respectively, to make the null phase frequency of each ring almost the same [21]. Each graphene-based HIS unit can be switched between its high-impedance and low-impedance states.

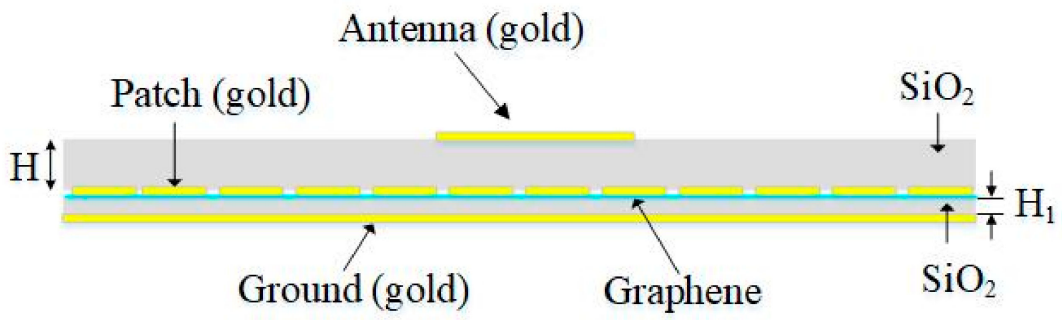

Figure 1. Side view of the loop antenna with the graphene-based high impedance surface (HIS).

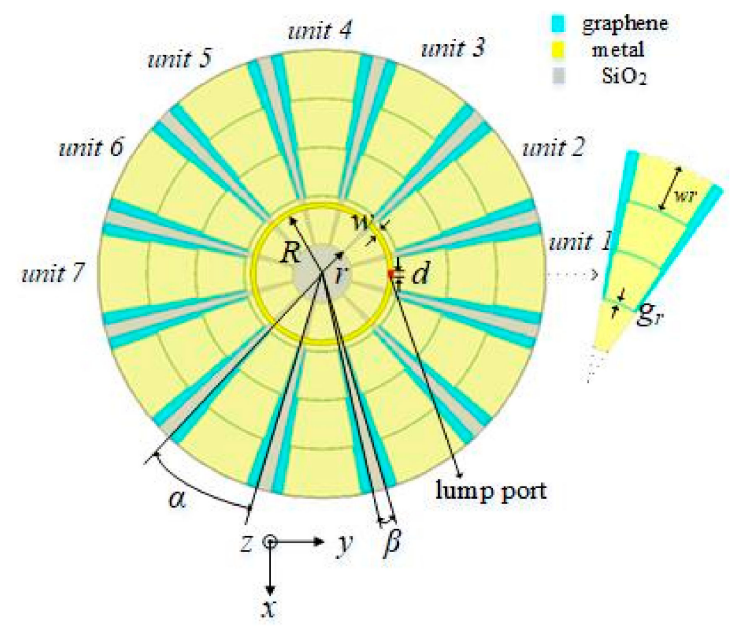

Figure 2. Top view of the loop antenna with the graphene-based high impedance surface (HIS).

The other layer of $\mathrm{SiO}_{2}$ with a thickness of $H=35 \mu \mathrm{m}$ is deposited on the HIS, and height $H$ has an important effect on the reflection coefficients and gains of the antenna. The loop antenna with radius $\mathrm{R}=60 \mu \mathrm{m}$ is deposited on the $\mathrm{SiO}_{2}$ substrate. The perimeter of the loop antenna equals approximately to $0.95 \lambda$ ( $\lambda$ is the guided wavelength at $0.5 \mathrm{THz}$ ). The numerical analysis software Ansoft HFSS (High Frequency Structure Simulator) is used to simulate the configuration. The graphene can be modelled by the impedance boundary, and we can set the value of the resistance and reactance, respectively. A $50 \Omega$ ideal wave source is used to feed the antenna. The dimensions of wave source $d$ and line width 
$w$ are 4 and $2 \mu \mathrm{m}$, respectively. Detailed techniques to fabricate these kinds of graphene antennas can be found in Reference [27,30], supporting the feasibility of our design.

\section{Results and Discussion}

To demonstrate the antenna operation performance, several different states of the loop antenna based on the switched HIS are simulated. For convenience of description, the number of high-impedance unit cells can be represented by $m(m=0,3,7,12)$. Take $\varphi=0^{\circ}$ plane as an example, when $m=0$, all of the graphene-based HIS units are set to low-impedance state, the beam direction is $\theta=0^{\circ}$. When $m=$ 3 or 7 , in the case that HIS units 3-5 or 1-7 in the upper plane are high-impedance states, the beam direction is $\theta=+5^{\circ}$ or $\theta=+10^{\circ}$. Symmetrically, the beam directions can tilt to $\theta=-5^{\circ}$ or $\theta=-10^{\circ}$. It is obvious that in case of $m=12$, the beam is also $\theta=0^{\circ}$, but the antenna gain increases compared to $m=$ 0 . Figure 3 shows the simulated radiation patterns on $\varphi=0^{\circ}$ plane with different high impedance unit cells. The antenna can realize a $\pm 10^{\circ}$ beam-tilt on $\varphi=0^{\circ}$ plane, and the beam-titled angle is smaller when the number of HIS units in high-impedance state is reduced.

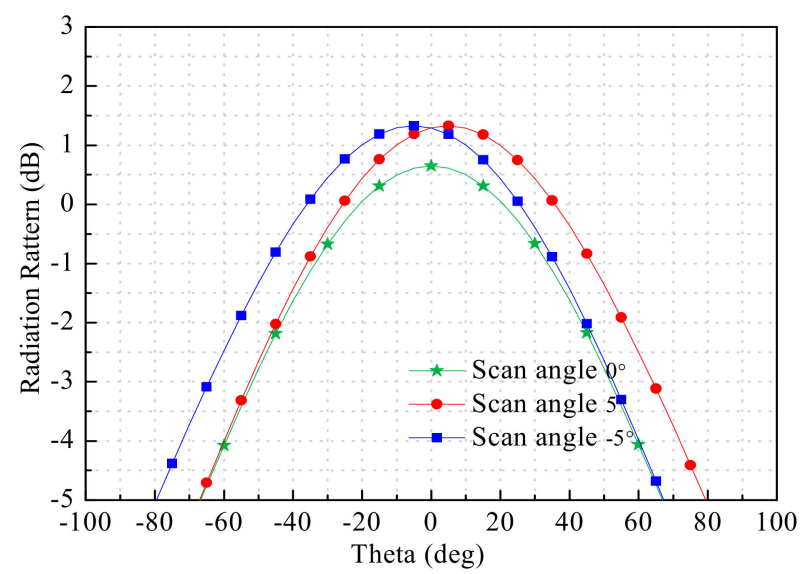

(a)



(b)

Figure 3. Simulated radiation patterns ( $\varphi=0^{\circ}$ plane) with different high impedance units at $0.5 \mathrm{THz}$. (a) $\theta=0^{\circ}(m=0)$ and $\theta= \pm 5^{\circ}(m=3)$. (b) $\theta=0^{\circ}(m=12)$ and $\theta= \pm 10^{\circ}(m=7)$.

To illustrate the working mechanism, Figure 4 shows the current distribution in case of $m=7$. We can see that the current distribution changes with the different HIS unit states. It can be seen from Figure $4 \mathrm{a}$ that when the HIS units 1-7 in the upper plane are in high-impedance states, the current is mainly distributed on the loop antenna and the HIS units 7-12. On the contrary, as shown in Figure 4b, when the HIS units 7-12 in the lower half plane are high-impedance states, the current is mainly distributed on the loop antenna and the HIS units 1-7. It can be found that the current is mainly 
distributed on the loop antenna and half of the graphene-based HIS units in the low-impedance state. Taking into account symmetry of the antenna, similarly, the antenna can also be capable of steering its beam from $-10^{\circ}$ to $+10^{\circ}$ on $\varphi=30^{\circ}, 60^{\circ}, 90^{\circ}, 120^{\circ}, 150^{\circ}$ plane. Figure 5 shows the definitions of the beam-steering angles $(\theta$ and $\varphi$ ). For example, when HIS units 4-6 or 2-8 are high-impedance states, the beam direction is $\theta=5^{\circ}$ or $\theta=+10^{\circ}$ on $\varphi=30^{\circ}$ plane, as shown in Figures 6 and 7.

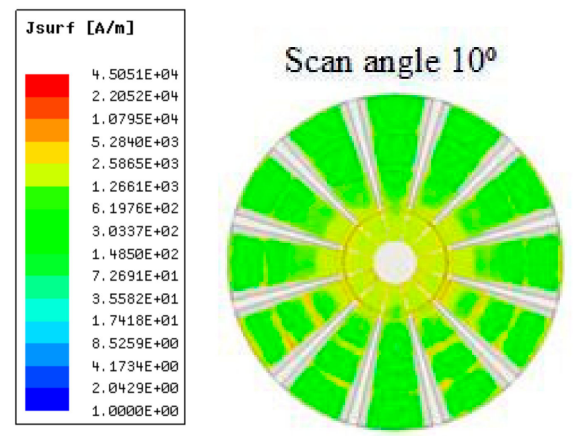

(a)

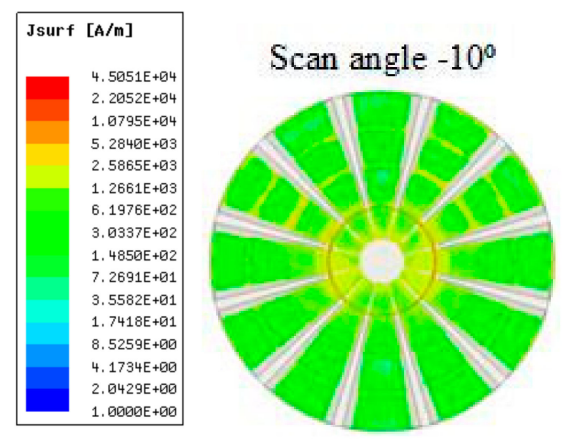

(b)

Figure 4. Simulated current distribution at $0.5 \mathrm{THz}(m=7)$, (a) The units $1-7$ are high-impedance states; (b) The units 7-12 are high-impedance states.



Figure 5. The beam-steering capabilities of the antenna $\theta \in\left\{0^{\circ}, \pm 5^{\circ}, \pm 10^{\circ}\right\} ; \varphi \in\left\{0^{\circ}, 30^{\circ}, 60^{\circ}, 90^{\circ}\right.$, $\left.120^{\circ}, 150^{\circ}\right\}$.

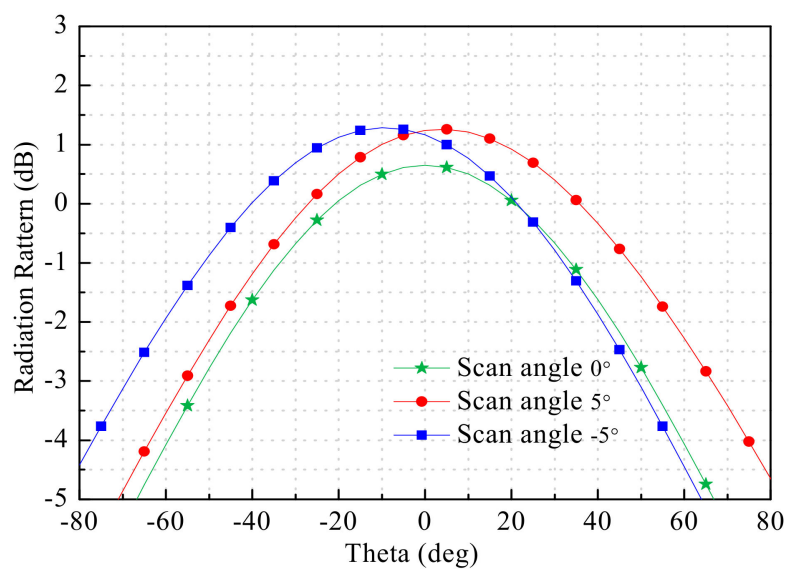

Figure 6. Simulated radiation patterns $\left(\varphi=30^{\circ}\right.$ plane $) \theta=0^{\circ}(m=0)$ and $\theta= \pm 5^{\circ}(m=3)$ at $0.5 \mathrm{THz}$. 


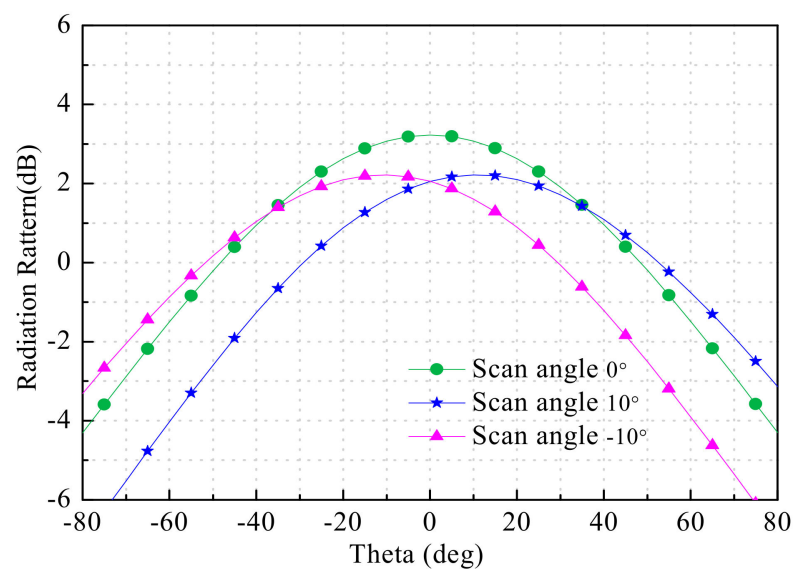

Figure 7. Simulated radiation patterns $\left(\varphi=30^{\circ}\right.$ plane $) \theta=0^{\circ}(m=12)$ and $\theta= \pm 10^{\circ}(m=7)$ at $0.5 \mathrm{THz}$.

The reflection coefficients of the antenna are shown in Figure 8. The $S_{11}$ becomes lower as $\mathrm{m}$ increases. Therefore, more high-impedance units are beneficial to the antenna impedance matching. Figure 9 shows the simulated reflection coefficients of the loop antennas with different radius over circular HIS, which indicates that the resonant frequency decreases with radius $R$ increasing. Also, it is obvious that the antenna can achieve beam steering in all these frequencies. The gains of the antenna are shown in Figure 10, the simulated peak gains in different states of $m=0,3,7,12$ at $0.5 \mathrm{THz}$ are 0.65, $1.33,1.99$, and $3.23 \mathrm{dBi}$, respectively.

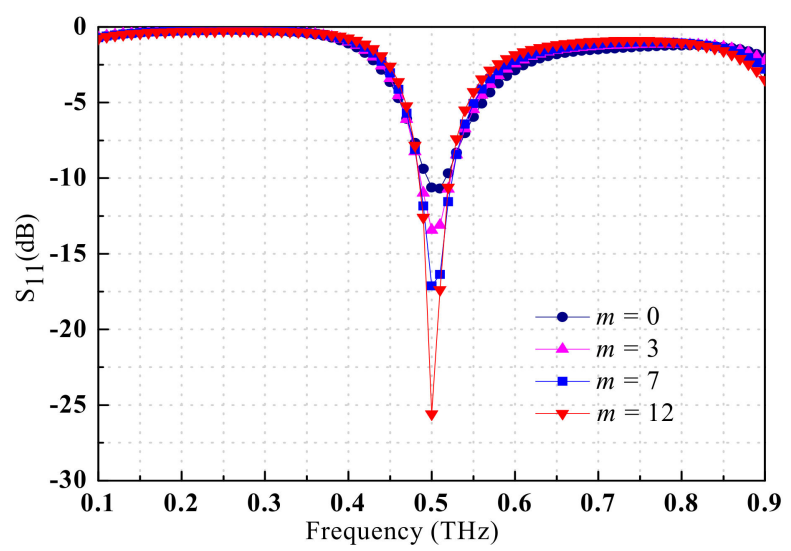

Figure 8. Simulated reflection coefficients with different high impedance units.

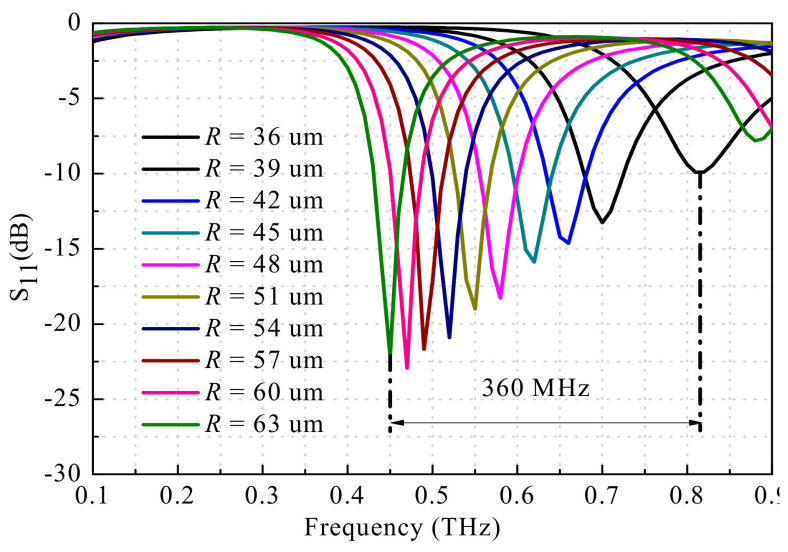

Figure 9. Simulated reflection coefficients of the loop antennas with different radius. 


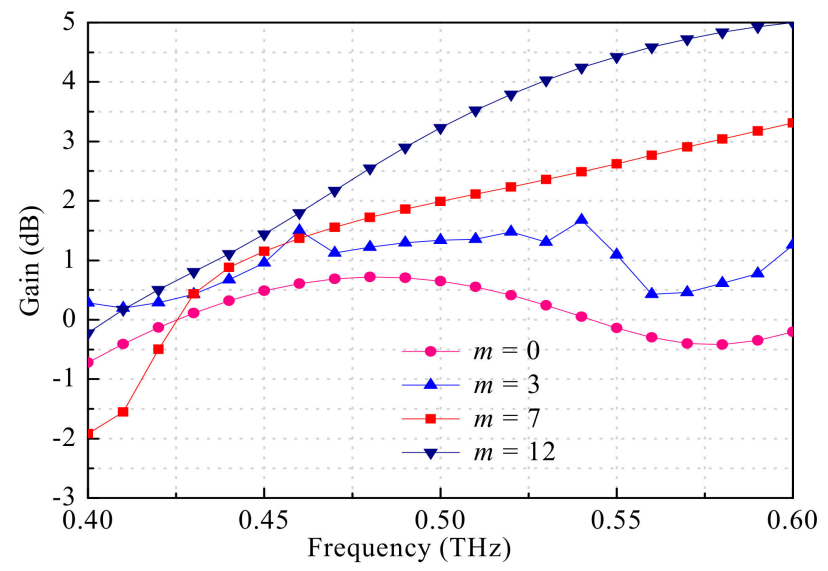

Figure 10. Simulated gains with different high impedance units.

Finally, Table 1 lists the key data of the proposed and those reported graphene antennas. Although the beam tilt angle in the elevation plane is relatively slighter, the proposed antenna can achieve beam steering in both the elevation and the azimuth plane.

Table 1. Performance of the proposed graphene antenna.

\begin{tabular}{ccccc}
\hline Ref. & $\begin{array}{c}\text { Resonant } \\
\text { Frequency (THz) }\end{array}$ & Gain (dBi) & $\begin{array}{c}\text { Beam Scan } \\
\text { Angle }\left(^{\circ}\right)\end{array}$ & $\begin{array}{c}\text { Azimuth Plane } \\
\text { Scan }\end{array}$ \\
\hline$[6]$ & 1.2 & 5.6 & $(-150-150)$ & \\
{$[19]$} & 1.1 & 1.08 & $(0-360)$ & \\
{$[20]$} & 2.6 & 4 & $(-70-70)$ & \\
{$[27]$} & 0.9 & 4 & $(-30-30)$ & $\sqrt{ }$ \\
Proposed & 0.5 & 3.2 & $(-10-10)$ & \\
\hline
\end{tabular}

\section{Conclusions}

In this paper, a novel 3D multi-beam steering $\mathrm{THz}$ loop antenna based on the switchable circular HIS is proposed. The antenna can achieve beam steering in both the elevation and the azimuth plane. Switching the states of the different graphene-based HIS unit cells between the high and low-impedance, the antenna is capable of steering $\mathrm{THz}$ main beam in 25 directions $\left(\theta \in\left\{0^{\circ}, \pm 5^{\circ}\right.\right.$, $\left.\left.\pm 10^{\circ}\right\} ; \varphi \in\left\{0^{\circ}, 30^{\circ}, 60^{\circ}, 90^{\circ}, 120^{\circ}, 150^{\circ}\right\}\right)$. Furthermore, the proposed design may be also applicable to other complex graphene-based wireless communication systems and graphene-based $\mathrm{THz}$ devices for different applications.

Author Contributions: Conceptualization, C.W. and Y.Y.; methodology, C.W.; software, C.W.; validation, C.W., Y.Y., J.Y. and X.C.; formal analysis, C.W.; investigation, C.W.; resources, C.W.; data curation, C.W.; writing-original draft preparation, C.W.; writing-review and editing, J.Y. and X.C.; visualization, C.W.; supervision, Y.Y.; project administration, Y.Y.; funding acquisition, Y.Y.

Funding: This research was funded by National Natural Science Foundation, grant number 61474112.

Acknowledgments: This work was funded by china scholarship council.

Conflicts of Interest: The authors declare that there is no conflict of interests regarding the publication of this article.

\section{References}

1. Han, L.C.X.; Wang, C.; Chen, X.; Zhang, W. Compact Frequency Reconfigurable Slot Antenna for Wireless Applications. IEEE Antennas Wirel. Propag. Lett. 2016, 15, 1795-1798. [CrossRef]

2. Majid, H.A.; Rahim, M.K.A.; Hamid, M.R.; Ismail, M.F. Frequency and Pattern Reconfigurable Slot Antenna. IEEE Trans. Antennas Propag. 2014, 62, 5339-5343. [CrossRef] 
3. Chen, Y.; Ye, L.; Zhuo, J.; Liu, Y.; Zhang, L.; Zhang, M.; Liu, Q.H. Frequency Reconfigurable Circular Patch Antenna with an Arc-Shaped Slot Ground Controlled by PIN Diodes. Int. J. Antennas Propag. 2017, 2017, 1-7. [CrossRef]

4. Trinh, L.H.; Le, T.N.; Staraj, R.; Ferrero, F.; Lizzi, L. A pattern-reconfigurable slot antenna for IoT network concentrators. Electronics 2017, 6, 105. [CrossRef]

5. Buttazzoni, G.; Comisso, M.; Cuttin, A.; Fragiacomo, M.; Vescovo, R.; Gatti, R.V. Reconfigurable phased antenna array for extending cubesat operations to Ka-band: Design and feasibility. Acta Astronaut. 2017, 137, 114-121. [CrossRef]

6. Luo, Y.; Zeng, Q.; Yan, X.; Wu, Y.; Lu, Q.; Zheng, C.; Hu, N.; Xie, W.; Zhang, X. Graphene-Based Multi-Beam Reconfigurable THz Antennas. IEEE Access 2019, 7, 30802-30808. [CrossRef]

7. Wu, L.; Qu, M.; Liu, Y. A generalized lossy transmission-line model for tunable graphene-based transmission lines with attenuation phenomenon. Sci. Rep. 2016. [CrossRef]

8. Xu, Z.; Dong, X.; Bornemann, J. Design of a Reconfigurable MIMO System for THz Communications Based on Graphene Antennas. IEEE Trans. Terahertz Sci. Technol. 2014, 4, 609-617. [CrossRef]

9. Dong, Y.; Liu, P.; Yu, D.; Li, G.; Tao, F. Dual-Band Reconfigurable Terahertz Patch Antenna With Graphene-Stack-Based Backing Cavity. IEEE Antennas Wirel. Propag. Lett. 2016, 15, 1541-1544. [CrossRef]

10. Tamagnone, M.; Gómez-Díaz, J.S.; Mosig, J.R.; Perruisseau-Carrie, J. Analysis and design of terahertz antennas based on plasmonic resonant graphene sheets. J. Appl. Phys. 2012, 112. [CrossRef]

11. Tamagnone, M.; Gómez-Díaz, J.S.; Mosig, J.R.; Perruisseau-Carrie, J. Reconfigurable terahertz plasmonic antenna concept using a graphene stack. Appl. Phys. Lett. 2012. [CrossRef]

12. Farmani, A.; Miri, M.; Sheikhi, M.H. Design of a high extinction ratio tunable graphene on white graphene polarizer. IEEE Photonics Technol. Lett. 2018, 30, 153-156. [CrossRef]

13. Fuscaldo, W.; Burghignoli, P.; Baccarelli, P.; Galli, A. Graphene Fabry-Perot Cavity Leaky-Wave Antennas: Plasmonic Versus Nonplasmonic Solutions. IEEE Trans. Antennas Propag. 2017, 65, 1651-1660. [CrossRef]

14. Gómez-Díaz, J.S.; Perruisseau-Carrier, J. Graphene-based plasmonic switches at near infrared frequencies. Opt. Express 2013, 21, 15490-15504. [CrossRef] [PubMed]

15. Sensale-Rodriguez, B.; Yan, R.; Kelly, M.M.; Fang, T.; Tahy, K.; Hwang, W.S.; Jena, D.; Liu, L.; Xing, H.G. Broadband graphene terahertz modulators enabled by intraband transitions. Nat. Commun. 2012, 3. [CrossRef] [PubMed]

16. Habibpour, O.; He, Z.S.; Strupinski, W.; Rorsman, N.; Ciuk, T.; Ciepielewski, P.; Zirath, H. Graphene FET Gigabit ON-OFF Keying Demodulator at 96 GHz. IEEE Electron Device Lett. 2016, 37, 333-336. [CrossRef]

17. Saeed, M.; Hamed, A.; Wang, Z.; Shaygan, M.; Neumaier, D.; Negra, R. Metal-Insulator-Graphene Diode Mixer Based on CVD Graphene-on-Glass. IEEE Electron Device Lett. 2018, 39, 1104-1107. [CrossRef]

18. Hamed, A.; Habibpour, O.; Saeed, M.; Zirath, H.; Negra, R. W-Band Graphene-Based Six-Port Receiver. IEEE Microw. Wirel. Components Lett. 2018, 28, 347-349. [CrossRef]

19. Cheng, X.; Wu, Y.; Yu, J.; Qu, S.-W.; Yao, Y.; Chen, X. Circular beam-reconfigurable antenna base on graphene-metal hybrid. Electron. Lett. 2016, 52, 494-496. [CrossRef]

20. Liang, F.; Yang, Z.-Z.; Xie, Y.-X.; Li, H.; Zhao, D.; Wang, B.-Z. Beam-Scanning Microstrip Quasi-Yagi-Uda Antenna Based on Hybrid Metal-Graphene Materials. IEEE Photon-Technol. Lett. 2018, 30, 1127-1130. [CrossRef]

21. Sarrazin, J.; Lepage, A.; Begaud, X. Circular High-Impedance Surfaces Characterization. IEEE Antennas Wirel. Propag. Lett. 2012, 11, 260-263. [CrossRef]

22. Vallecchi, A.; De Luis, J.R.D.; Capolino, F.; De Flaviis, F. Low profile fully planar folded dipole antenna on a high impedance surface. IEEE Trans. Antennas Propag. 2012, 60, 51-62. [CrossRef]

23. Amiri, M.A.; Balanis, C.A.; Birtcher, C.R. Analysis, design, and measurements of circularly symmetric high-impedance surfaces for loop antenna applications. IEEE Trans. Antennas Propag. 2016, 64, 618-629. [CrossRef]

24. Li, M.; Xiao, S.-Q.; Wang, Z.; Wang, B.-Z. Compact Surface-Wave Assisted Beam-Steerable Antenna Based on HIS. IEEE Trans. Antennas Propag. 2014, 62, 3511-3519. [CrossRef]

25. Mohamad, S.; Cahill, R.; Fusco, V. Selective High Impedance Surface Active Region Loading of Archimedean Spiral Antenna. IEEE Antennas Wirel. Propag. Lett. 2014, 13, 810-813. [CrossRef]

26. Chen, Y.-S.; Ku, T.-Y. A low-profile wearable antenna using a miniature high impedance surface for smartwatch applications. IEEE Antennas Wirel. Propag. Lett. 2016, 15, 1144-1147. [CrossRef] 
27. Huang, Y.; Wu, L.-S.; Tang, M.; Mao, J. Design of a Beam Reconfigurable THz Antenna With Graphene-Based Switchable High-Impedance Surface. IEEE Trans. Nanotechnol. 2012, 11, 836-842. [CrossRef]

28. Wang, X.-C.; Zhao, W.-S.; Hu, J.; Yin, W.-Y. Reconfigurable terahertz leaky-wave antenna using graphene-based high-impedance surface. IEEE Trans. Nanotechnol. 2015, 14, 62-69. [CrossRef]

29. Huang, Y.; Wu, L.-S.; Mao, J. Design of a switchable high impedance surface based on single-layer doped graphene for THz application. In Proceedings of the 2011 International Conference on Electromagnetics in Advanced Applications, Torino, Italy, 12-16 September 2011. [CrossRef]

30. Carrasco, E.; Tamagnone, M.; Perruisseau-Carrier, J. Tunable graphene reflective cells for THz reflectarrays and generalized law of reflection. Appl. Phys. Lett. 2013, 102. [CrossRef]

(C) 2019 by the authors. Licensee MDPI, Basel, Switzerland. This article is an open access article distributed under the terms and conditions of the Creative Commons Attribution (CC BY) license (http://creativecommons.org/licenses/by/4.0/). 